\title{
On the outbursts of black hole soft X-ray transients
}

\author{
Ü. $\operatorname{Ertan}^{1}$ and M. A. Alpar ${ }^{2}$ \\ 1 Physics Department, Middle East Technical University Ankara 06531, Turkey \\ 2 Sabancı University, Orhanl1-Tuzla 81474 İstanbul/ Turkey \\ e-mail: alpar@sabanciuniv.edu.tr
}

Received 4 March 2002 / Accepted 4 July 2002

\begin{abstract}
We suggest a new scenario to explain the outburst light curves of black hole soft X-ray transients together with the secondary maximum and the bump seen on their decay phases. Our explanations are based on the disk instability models considering the effect of X-ray irradiation. The scenario is consistent with the observed X-ray delays by a few days with respect to the optical rise for both the main outburst and the later maxima. We test our scenario by numerically solving the disk diffusion equation. The obtained model curve fits well to the observed X-ray outburst photon flux curve of the black hole soft X-ray transient GS/GRS 1124-68, a typical representative of the four BH SXTs with very similar light curves.
\end{abstract}

Key words. accretion - accretion disks - instabilities - stars: binaries: cose - X-rays: general

\section{Introduction}

Soft X-ray transients (SXTs), a subclass of low mass X-ray binaries, contain either a neutron star (NS SXTs) or a black hole (BH SXTs). Their sporadic outbursts with observed or estimated recurrence time scales changing from months to more than 50 years show a variety of light curves (Chen et al. 1997). Among these sources the BH SXTs GRO J0422+32, A062000, GS/GRS 1124-68 and GS 2000+25 show very similar $\mathrm{X}$-ray outburst light curves. Rise to maximum is fast (few days). Decay can be fitted with exponentials, or with power laws. These four sources are usually labeled as FRED (fastrise-exponential-decay) sources. We refrain from using the term FRED because the initial decays can also be fitted with power laws. Instead, we shall use the term BH SXTs to cover only these four sources and the term "exponential-like" to describe their similar secular decay behaviors. Other common features of the outburst light curves are a typical secondary maximum seen in the decay phase about two months after the main maximum and a bump seen at the end of the decay phase, after the secondary maximum. The secondary maximum is exhibited by all four BH SXTs above. The bump is seen in the outburst light curves of A 0620-00 and GS/GRS 1124-68. It is not very clear in the light curve of GS $2000+25$ and absent in that of GRO J0422+32 (Tanaka \& Shibazaki 1996).

Three physical effects are likely to determine the behavior of SXTs. These effects are (i) viscous evolution of the accretion disk, (ii) transitions between the two stable states of the disk through hydrogen ionization, as in disk instability models, and

Send offprint requests to: $\ddot{U}$. Ertan,

e-mail: unal@astroa.physics.metu.edu.tr (iii) irradiation of the disk by X-rays from the inner disk (also from the neutron star surface in the case of NS SXTs). In this paper we present a comprehensive model for the black hole SXTs based on an interplay between all three effects.

The disk instability models (DIMs) which are successful in reproducing the general characteristics of dwarf nova (DN) light curves (Osaki 1974; Hoshi 1979; Meyer \& MeyerHofmeister 1981) are also suggested to be the possible mechanism for the SXT outbursts (Cannizzo et al. 1985; Huang \& Wheeler 1989; Mineshige \& Wheeler 1989). According to DIMs the equilibrium solutions on the accretion rate $\dot{M}$ - surface density $\Sigma$ plane form an "S" shaped curve for a given radial distance $\mathrm{R}$ from the center of the disk. The upper and lower branches of the curve are viscously stable, whereas the middle branch is unstable. The unstable branch appears due to partial ionization of hydrogen and corresponds roughly to the temperature range $T_{\text {eff }} \sim 6 \times 10^{3}-10^{4} \mathrm{~K}$. According to DIMs all the disk is in the cold stable branch during the quiescent state. In the cold stable regime, surface densities increase with time at all radii due to the low accretion efficiency. The critical maximum surface density of the cold branch is eventually exceeded first at some particular radius $R$, and the instability propagates as a density wave towards the smaller and the larger radii. If all or most of the disk jumps to the hot stable branch the increasing accretion rate leads to an outburst. For the disk to return to the quiescent phase the surface density should decrease below the critical minimum surface density of the hot stable branch at some location in the disk. This is expected to occur first at the outermost regions of the disk, where in the hot state the surface densities are lowest, while the critical surface densities are highest. Consequently, all the disk returns back to the cold 
stable regime by means of a cooling front propagating from the outer disk towards the inner regions. The observed decay in luminosities subsequent to a burst is expected to be driven by the propagation of this cooling front.

The standard version of DIMs have difficulties in explaining the long recurrence times and the slow decay characteristic of the SXT outbursts with plausible standard thin disk $\alpha$ parameters (Shakura \& Sunyaev 1973) estimated from DN light curve analyses. These difficulties do not exclude the DIMs as the mechanism for the SXT outbursts, but require a modification of their standard form. Including the evaporation and the consequent disk truncation in the models it is possible to explain the long recurrence time scales (few decades or more) of $\mathrm{BH}$ SXTs (Meyer-Hofmeister \& Meyer 1999; Dubus et al. 2001). $\mathrm{X}$-ray irradiation of the outer disk and possibly the evaporation also seem to be responsible for the observed characteristics of the decay curves of BH SXTs. It has long been known that the X-ray irradiation is the dominant source of the observed optical light of persistent LMXBs and in particular SXTs in their outburst states. It was suggested that the X-ray irradiation may have a stabilizing effect on the outer mass flow keeping the outer disk temperatures above the hydrogen ionization temperature (Meyer \& Meyer-Hofmeister 1984; van Paradijs $\&$ McClintock 1994). In a previous work, we showed that pure viscous evolution of matter located originally at the outer disk can reproduce the rise, the turnover and the early decay characteristics of the BH SXTs by fitting the model light curves to the observed X-ray photon flux data of the BH SXTs GS/GRS 1124-68 and GS 2000+25 (Ertan \& Alpar 1998). This also suggests the importance of the X-ray irradiation, since the irradiation could build up the conditions for a pure viscous decay by preventing the propagation of a cooling front for long times. By analytical treatments, King \& Ritter (1998) (KR) concluded that the slow decay is produced when the disk is fully ionized by irradiation, for inner accretion rates above a certain critical $\dot{M}_{\text {in }}$ and the decay seemingly linear in time that is seen at the end of the decay phase is produced below this $\dot{M}_{\text {in }}$, when a cooling front propagates inwards from the outer disk. Detailed numerical calculations show that a disk fully ionized by strong irradiation gives a much slower decay than the observed decays (Cannizzo 2000). By adjusting the evaporation rate in his model, Cannizzo (2000) obtained the observed decay behavior for a fully ionized disk. On the other hand, following the idea of KR, but employing a moderate irradiation in models, it is also possible to reproduce the observed decays with a negligible effect of evaporation on the decay curves (Dubus et al. 2001; DHL).

The vertical structure analyses of an X-ray irradiated disk show that the direct illumination of the outer disk by the central X-rays is not possible due to the self screening of the disk (Dubus et al. 1999). On account of the fact that the observations invariably give much higher $L_{\mathrm{opt}} / L_{\mathrm{x}}$ ratio than expected from intrinsic dissipation alone, Dubus et al. (1999) suggested that the X-ray illumination is very probably still present, but indirect or the outer disk is warped. Indirect irradiation of the outer disk may take place via scattering. Even if the inner disk is vertically optically thick and geometrically thin, the surface layers where heating dominates over cooling could be thermally unstable and could evaporate to a hot corona (Shaviv \& Wehrse 1986). Existence of such a hot corona surrounding the inner disk will be our main assumption regarding the mechanism of indirect irradiation.

Since the optical flux is X-ray irradiation dominated, a change in the intrinsic dissipation at the outer disk can hardly be the reason for the observed enhancement in optical flux at the secondary maximum and the bump. Irradiation must be incorporated in the explanation of these features. In an alternative scenario tidal instabilities due to the 3:1 resonance (Frank et al. 1992) at the outer disk could modify the optical light of the BH SXTs, but only if the disk size increases considerably, intercepting more of the X-rays coming from the inner disk (Haswell et al. 2000). However, it is unlikely that the consequent changes in the mass inflow rate in the outer disk can lead to the observed secondary maximum or the bump.

A further restriction on models comes from the comparison of the optical and X-ray observations. Detailed analyses show that whenever it is possible to make a clear comparison between the optical and the X-ray observations of the main and the minor maxima of BH SXTs, it is seen that the optical rise precedes the X-ray rise. The X-ray delays with respect to the optical light are $\sim 4-6$ days for the main and the secondary maximum and around two weeks for the bump (Kuulkers 1998; Ebisawa et al. 1994; Orosz et al. 1997). Considering these observational constraints, we suggest an explanation for the overall outburst phase of BH SXTs including the secondary maximum (Sect. 2) and the bump (Sect. 3). The details of the numerical model testing these explanations is presented in Sect. 4. In Sect. 5 we summarize and discuss the results.

\section{The main outburst and the secondary maximum}

The secondary maximum is seen roughly two months after the main maximum. An abrupt increase in the X-ray light curve by a factor $\sim 1.5-2$ is followed by a curve that remains roughly parallel to the extrapolation from the main decay in the logarithmic plot (Tanaka \& Shibazaki 1996).

Mineshige (1994) pointed out that an abrupt heating of the disk and a substantial mass supply are required to account for the observed secondary maximum, and attributed the onset of the X-ray heating of the disk to removal of a Compton cloud, which had been blocking the central X-rays from reaching the outer parts. He suggested that the mass supply is either due to an enhanced mass transfer from the companion or a transient recession of the cooling front in the disk. Some difficulties arise in either case. Mass transfer from the companion is unlikely, since the $L_{1}$ point is shielded by the disk. Even if the $L_{1}$ point is irradiated it is extremely difficult to increase the mass transfer rate by the X-ray heating unless the X-ray spectrum is very hard (King 1989). On the other hand, if X-ray irradiation were blocked by a central corona, then a cooling front would have propagated in the two months before the occurrence of the secondary maximum. In that case the optical luminosity would decay faster than the X-ray luminosity which is not the case.

Chen et al. (1993) explained both the secondary maximum and the bump by an enhanced mass transfer rate from the companion star. They suggested that the secondary maximum is 
due to the heating of the outer layers of the atmosphere of the secondary when the outer disk becomes optically thin while the bump occurs via a mass transfer instability caused by hard $\mathrm{X}$-ray heating of the subphotospheric layers of the secondary during the main outburst. They attributed the delay between the secondary maximum and the bump to the transport of part of absorbed hard X-ray energy to the entire convective region of the secondary by convection. It seems hard to account for the abrupt rise of the mini outbursts by these mechanisms. In addition, if the $L_{1}$ point is indeed illuminated because of the evaporation of the outer disk then X-ray eclipses would be observed in sources with moderate to high inclination. X-ray eclipses were not seen during the decay phase of the secondary maximum from any of our four sources.

Since the observed optical light is likely to be X-ray irradiation dominated, the optical rise during the secondary maximum is expected to occur due to either an increase in the central X-ray luminosity or a change in the outer disk properties, thereby intercepting a larger fraction of the X-rays. Since the observed optical rise precedes the X-ray rise by a few days, the former possibility is not favorable. At the beginning of the main outburst, the accumulated matter at the outer disk expands to both smaller and larger radii. The effective X-ray heating of the outer disk cannot start immediately upon the triggering of the outburst. This takes a viscous time scale corresponding to the expansion of the released matter from the outer to the inner disk. In this time the outer disk may already have gone into the cold stable state by means of a cooling front propagation before the central X-ray luminosity affects the stability of the outer disk. KR suggested that the X-ray irradiation can cause this outermost cold disk to jump back to the hot stable branch at the very beginning of the outburst and increase the amplitude of the X-ray luminosity about $\sim 40-120$ days after the main $\mathrm{X}$-ray maximum. In addition, they pointed out that the optical flux cannot rise until after the increased mass flow reaches the inner disk and increases the X-ray radiation. This is contradicted by the observations. This suggestion was not confirmed by detailed numerical model calculations (Cannizzo 1998; Dubus et al. 2001). By comparing the results of nonirradiated and irradiated disk models with the observations, Cannizzo (1998) concluded that a completely and strongly irradiated disk model overestimates the optical flux at the maximum light of A0620-00, and that the irradiation temperatures $T_{\text {irr }} \simeq 0.3-0.4 T_{\text {eff }}$ at the outer disk are enough to explain the observed optical flux if all the disk is irradiated. Incorporating effects of evaporation in fully irradiated and completely ionized disk models, Cannizzo (2000) showed that the evacuation of the inner disk due to the strong evaporation, and the subsequent refilling could reproduce the observed features of both the main decay and the secondary maximum. Note, however, that this model does not address the observed X-ray delays.

We now turn to the second option, a change in the outer disk properties. We propose that the part of the outer disk that made the transition to the cold state before the increase of the central accretion rate associated with the main outburst may not be heated by the central X-rays for a while after the main outburst, even when the X-ray luminosity reaches its maximum. This is because the pressure scale height of the outermost cold disk should be lower than that of the intermediate disk, at radii less than and of the order of a "hot disk radius" $R_{\mathrm{h}}$. To put this in other words, the cooling front propagating inwards is stopped at a radius $R_{\mathrm{h}}$ by the increasing $\mathrm{X}$-ray luminosity, and the outer disk beyond $R_{\mathrm{h}}$ remains shielded and cold. The cooling front at $R=R_{\mathrm{h}}$ cannot propagate inwards until the irradiation temperatures at $R \lesssim R_{\mathrm{h}}$ drop below the hydrogen ionization temperatures. We shall see from the model fits that $R_{\mathrm{h}}$ actually remains roughly constant during the early decay phase. $R_{\mathrm{h}}$ separates regions of different evolution: The hot matter inside $R_{\mathrm{h}}$ depletes faster than the cold matter outside $R_{\mathrm{h}}$, due to the high viscosities operating in the hot state.

The shielding is removed naturally when the thickness $h$ at $R \lesssim R_{\mathrm{h}}$ decreases enough for the central $\mathrm{X}$-rays to illuminate a part of the cold disk at $R>R_{\mathrm{h}}$. If the $\mathrm{X}$-ray irradiation is still high enough to trigger a thermal-viscous instability at the outermost cold disk then the resultant disk evolution can account for the observed properties of the secondary maximum. The increasing illuminated disk area (because of the removal of the shielding of the outermost disk) leads first to the observed optical rise. This is followed by an enhanced rate of mass inflow. This scenario can account for the observed X-ray delay by a few days, corresponding to the viscous time scale for matter released from the outermost disk to accrete to the inner disk.

In the numerical calculations, we simply remove the shielding at a parametrized time $t_{1}$ and see that the consequent model $\mathrm{X}$-ray photon flux curve fits well to the data. With our numerical calculations we present disk thickness $h(R)$ profiles, for different times of the main decay before the onset of the secondary maximum, calculated using the midplane temperatures of our 1-D model. These results indicate that, in the 1-D model, the disk does evolve to remove the shielding.

\section{The bump}

Outburst light curves of the BH SXTs A0620-00 and GS/GRS 1124-68 also show a bump $\sim 150-170$ days after the main maximum. After the bump, the X-ray and the optical light curves decrease steeply and the system returns to quiescence (Tanaka \& Shibazaki 1996). Unlike the main outburst and the secondary maximum, the bump does not rise very rapidly. The rise time scale is about few weeks. The optical maximum precedes the X-ray maximum by about two weeks for A0620-00 (Kuulkers 1998). The onset of the bump occurs when the system is in the so called low-hard state.

Following the secondary maximum, the disk is again made of a cold outer region beyond $R_{\mathrm{h}}$, shielded by the hot, irradiated inner disk that prevails at $R \lesssim R_{\mathrm{h}}$. Like the secondary maximum, the bump is caused by irradiation reaching the outer disk and enhancing mass transfer. The different morphology of the bump indicates that in this instance, irradiation reaches the outer disk by a mechanism different from that causing the secondary maximum. The increase of the X-ray hardness ratio starts a few weeks before the onset of the bump. The hard X-ray photons are generally believed to be the up-scattered photons from a hot corona around the inner disk. A hot corona could be formed due to the thermal instabilities from the optically thin surface layers of the inner disk (Shaviv \& Wehrse 1986), 
and it could be stabilized by the soft photons coming from the photosphere of the underlying thin disk. The seed photons provide cooling for the hot corona by means of inverse Compton scattering. As the accretion into the inner geometrically thin disk decreases, the black-body temperature of the inner disk and hence the number of seed photons also decrease. The cooling becomes less effective and the temperature of the corona increases. We suggest that the consequent increase in the size of the corona increase the efficiency of the X-ray irradiation. A thermal viscous instability could be triggered when the corona reaches dimensions large enough to illuminate the outer disk beyond the radius $R_{\mathrm{h}}$. This leads to an increase first in the optical luminosity and then in the X-ray luminosity. The gradual expansion-illumination-ionization sequence is followed by the slow rise of the bump because of reduced viscosity and longer viscous time scales in this regime compared to the conditions that prevailed at the secondary maximum.

\section{The numerical model}

In the thin disk approximation with $\Omega \simeq \Omega_{\mathrm{K}}=\left(G M / R^{3}\right)^{1 / 2}$, the mass conservation equation

$R \frac{\partial \Sigma}{\partial t}+\frac{\partial}{\partial R}\left(R \Sigma V_{R}\right)=0$

the angular momentum conservation equation

$R \frac{\partial}{\partial t}\left(\Sigma R^{2} \Omega\right)+\frac{\partial}{\partial R}\left(R \Sigma V_{R} R^{2} \Omega\right)=\frac{1}{2 \pi} \frac{\partial g}{\partial R}$,

together with the expression for the torque

$g=2 \pi R v \Sigma R^{2}\left(\frac{\partial \Omega}{\partial R}\right)$

give a non-linear diffusion equation for the surface density

$\frac{\partial \Sigma}{\partial t}=\frac{3}{R} \frac{\partial}{\partial R}\left[R^{1 / 2} \frac{\partial}{\partial R}\left(v \Sigma R^{1 / 2}\right)\right]$.

With the assumption that $T_{\mathrm{c}}^{4} \gg T_{\mathrm{eff}}^{4}$, we can write

$\frac{4 \sigma}{3 \tau} T_{\mathrm{c}}^{4}=\frac{9}{8} \nu \Sigma \frac{G M}{R^{3}}=\sigma T_{\mathrm{eff}}^{4}$

where $T_{\mathrm{c}}$ and $T_{\text {eff }}$ are disk midplane and effective temperatures respectively, $\tau \sim \kappa_{R} \Sigma$ is the vertically integrated optical depth, and $v$ is the kinematic viscosity. We take the density $\rho=\Sigma / 2 h$ where $h$ is the pressure scale height of the disk and the temperature $T=T_{\mathrm{c}}$ to estimate the Rosseland mean opacities $\kappa_{R}$ by using the opacity tables for population I stars with mixture $X=0.7$ and $Z=0.02$ (Alexander \& Fergusson 1994, for $\log T \leq 3.7$ and Iglesias \& Rogers 1996, for $\log T>3.7$ ). For the viscosity we use the standard $\alpha$ prescription $v=\alpha c_{\mathrm{s}} h$ and adopt the commonly used bimodal $\alpha$ parameter. We set $\alpha=\alpha_{\mathrm{h}}=0.1$ and $\alpha=\alpha_{\mathrm{c}}=3.3 \times 10^{-2}$ for the hot and cold stable states respectively. We neglect the radiation pressure and take the local sound speed $c_{\mathrm{s}}=k T_{\mathrm{c}} / \mu m_{\mathrm{p}}$ where $k$ is the Boltzmann constant, $m_{\mathrm{p}}$ is the proton rest mass, and $\mu$ is the mean molecular weight. We set $\mu=0.63$ in the hot regime, and $\mu=0.87$ in the cold regime. Equation (5) assumes that the local viscously dissipated energy is instantaneously radiated from the disk photosphere in the form of black-body radiation.

By setting $x=2 R^{1 / 2}$ and $S=x \Sigma$, Eq. (4) can be written in a simple form

$\frac{\partial S}{\partial t}=\frac{12}{x^{2}} \frac{\partial^{2}}{\partial x^{2}}(v S)$

We divide the disk into equally spaced 200 grid points in $x$. This means that the grid spacing in $R$ space decreases with decreasing $R$. This is preferred for a better spatial resolution of the inner disk. Although our time steps are small enough $(\sim$ few seconds) for a good time resolution, 200 grid points in $x$ space provide a low space resolution. Increasing spatial resolution requires shorter time steps and extremely long computation times to scan the disk parameters to obtain a good fit to the observed X-ray photon flux curve of the BH SXT GS/GRS 1124-68. We therefore restrict the computation to 200 grid points in $x$ at present.

Since the GINGA ASM photon flux data (1-20 keV) is not de-convolved data, we convolve our model photon flux curve with the detector response matrix. The observed data and the response matrix is provided by $\mathrm{S}$. Kitamoto (private communication). After Eq. (6) is numerically solved, we further divide the main spatial grid at each time step into 20 equally spaced grid points in $x$ to determine the effective temperature distribution and the corresponding X-ray photon flux with a higher accuracy. We take the black hole mass $M_{1}=6 M_{\odot}$, the distance $d=3 \mathrm{kpc}$ (McClintock et al. 1992; West 1991). We neglect the neutral hydrogen absorption. The inclination angle was left as free parameter; $\cos i=0.88$ was obtained from our fits.

\subsection{X-ray irradiation}

The X-ray irradiation flux is given by

$\sigma T_{\mathrm{irr}}^{4}=\frac{\eta \dot{M}_{\mathrm{in}} c^{2}(1-\epsilon)}{4 \pi R^{2}}\left(\frac{H_{\mathrm{irr}}}{R}\right)^{n}\left(\frac{\mathrm{d} \ln H_{\mathrm{irr}}}{\mathrm{d} \ln R}-1\right)$

(Shakura \& Sunyaev 1973), where $\eta$ is the efficiency of the conversion of the rest mass energy into X-rays, $\epsilon$ is the $\mathrm{X}$-ray albedo of the disk face, $\sigma$ is the Stefan-Boltzmann constant, $H_{\text {irr }}$ is the local pressure scale height of the disk which should be calculated including the effect of X-ray irradiation itself (Dubus et al. 1999), and $\dot{M}_{\text {in }}$ is the inner accretion rate. Shakura \& Sunyaev (1973) estimated that $n=1$ for the neutron star systems, and $n=2$ for the black hole systems. King $\&$ Ritter (1998) pointed out that $n$ could be taken as unity for also the black hole systems, assuming that the source of the $\mathrm{X}$-ray irradiation is a hot corona around the inner disk rather than the thin inner disk surface. For a point source, Eq. (7) can be rewritten as

$\sigma T_{\mathrm{irr}}^{4}=C \frac{\dot{M}_{\mathrm{in}} c^{2}}{4 \pi R^{2}}$

where

$C=\eta(1-\epsilon) \frac{H_{\text {irr }}}{R}\left(\frac{\mathrm{d} \ln H_{\text {irr }}}{\mathrm{d} \ln R}-1\right)$. 
$C$ can vary in a large interval for different choices of the parameters especially due to the uncertainty on the X-ray albedo $\epsilon$. For a point source, estimates for $C$ are usually in the range $10^{-4}-10^{-3}$ (Tuchman et al. 1990; de Jong et al. 1996; Dubus et al. 1999) In our numerical model, we use Eq. (8) parametrizing $C$ to calculate the irradiation temperatures (see Sects. 4.4 and 4.5).

Detailed vertical disk structure analyses show that the $\mathrm{S}$ shaped characteristic of the equilibrium curves disappear above $T_{\text {irr }} \sim 10^{4} \mathrm{~K}$ and the disk can remain in a hot stable state (Tuchman et al. 1990; Dubus et al. 1999). In these analyses the X-rays are assumed to be absorbed in a thin layer of the disk surface. With this assumption, these results show that the radiative hot, optically thick disks are modified by the X-ray irradiation in the regions near the disk surface, while the regions near the disk midplane, where most of the matter is located, could remain unaffected. That is, an efficient X-ray irradiation can change the stability criteria of the hot radiative disks by changing the boundary conditions at the disk surface without modifying the disk midplane conditions. Following these results, in our numerical model which does not address the vertical disk structure we estimate the viscosities by using the disk midplane temperatures $T_{\mathrm{c}}$. The irradiation temperatures, on the other hand, are used to calculate the critical minimum and maximum surface densities at a given time at each grid point, and thereby to adjust the $\alpha$ parametrization (either $\alpha_{\mathrm{h}}=0.1$ or $\left.\alpha_{\mathrm{c}}=0.033\right)$ throughout the disk.

\subsection{Critical surface densities}

Various authors have obtained minimum and maximum critical surface densities of the hot and cold stable states for non irradiated and steady state disk accretion by performing numerical fits to the turning points of S-shaped equilibrium curves. Although the critical surface densities are model dependent, there is a close agreement between these results (Shafter et al. 1986; Cannizzo et al. 1988; Ludwig et al. 1994; Hameury et al. 1998).

Most recently Dubus et al. (2001) computed $70 \times 120 \times$ $190 \times 9$ vertical disk structures in $\Omega_{\mathrm{K}}, \Sigma, T_{\mathrm{c}}, T_{\text {irr }}$ with parameter ranges convenient for SXTs for both irradiated and nonirradiated disks. They found

$\Sigma_{\max }=(10.8-10.3 \xi) \alpha_{\mathrm{c}}^{-0.84} M_{1}^{-0.37+0.1 \xi} R_{10}^{1.11-0.27 \xi} \mathrm{g} \mathrm{cm}^{-2}$

$\Sigma_{\min }=(8.3-7.1 \xi) \alpha_{\mathrm{h}}^{-0.77} M_{1}^{-0.37} R_{10}^{1.12-0.23 \xi} \mathrm{g} \mathrm{cm}^{-2}$

where $\xi=\left(T_{\mathrm{irr}} / 10^{4} \mathrm{~K}\right)^{2}, M_{1}$ is the mass of the compact object in units of solar masses, and $R_{10}=\left(R / 10^{10} \mathrm{~cm}\right)$. These results provide an estimation of the critical surface densities for the irradiated unsteady disks, as well as for non-irradiated disks $(\xi=0)$. We adopt the critical surface densities given by the Eqs. (10) and (11) with $\alpha_{\mathrm{h}}=0.1$ and $\alpha_{\mathrm{c}}=0.033$.

\subsection{The initial mass distribution and the early rise phase}

We start with a Gaussian initial mass distribution at the beginning of the outburst phase

$\Sigma(R, t=0)=\Sigma_{0} \exp \left[-\left(\frac{R-R_{0}}{\Delta R}\right)^{2}\right]$

where $R_{0}=9.0 \times 10^{10} \mathrm{~cm}$ is the location of the center of the Gaussian , $\Delta R=2 \times 10^{10} \mathrm{~cm}$, and $\Sigma_{0}$ is the surface density at the center of the Gaussian at time $t=0$. The critical surface density $\Sigma_{\max }$ is found to be close to $\Sigma_{0}$ in our best fits. We iterate and choose a fit with $\Sigma_{0} \simeq \Sigma_{\max }$. This critical $\Sigma_{\max }$ is calculated for the non-irradiated case, since the quiescent X-ray luminosity is in operation when the instability is triggered. We obtain the best fits for $\Sigma_{0}=1.1 \times 10^{3} \mathrm{~g} \mathrm{~cm}^{-2}$ with chosen $R_{0}$. Substituting $\alpha_{\mathrm{c}}=0.033$, the black hole mass $M_{\mathrm{x}}=6 M_{\odot}$ and $R_{0}=9 \times 10^{10} \mathrm{~cm}$ in Eq. (10), we find $\Sigma_{\max }=1.1 \times 10^{3} \mathrm{~g} \mathrm{~cm}^{-2}=\Sigma_{0}$ for the non-irradiated case $(\xi=0)$. The calculation has several free parameters. These are $(i)$ the time $t_{0}$, when the X-ray irradiation starts - we found $t_{0}=3 \mathrm{~d}$. (ii) $T_{\text {eff,min }}$ above which the disk is taken to be in the hot state until $t=t_{0}$ (after $t=t_{0}$ the critical surface densities (Eqs. (10) and (11)) for an irradiated disk are used). (iii) The time $t_{1}$ at which the shielding is removed, leading to the secondary maximum. (iv) The time $t_{2}$ when the irradiation efficiency increases leading to the bump.

We start with the hot state viscosities $\left(\alpha=\alpha_{\mathrm{h}}\right)$. Until $t=t_{0}=3 \mathrm{~d}$ the radial grid points having $T_{\mathrm{eff}}>10000 \mathrm{~K}$ are kept in the hot state, and we set $\alpha=\alpha_{\mathrm{c}}$ for lower $T_{\text {eff }}$. We switch on the X-ray irradiation at $t \simeq 3 \mathrm{~d}$, and the outermost model disk having $\alpha=\alpha_{\mathrm{c}}$ at that moment is assumed to be shielded, and so remains in the cold state until $t=t_{1}$ at which we remove the shielding. To fix the given parameters (for the first three days), we follow the quality of the fits until the end of the overall decay phase for many trials. This is because the amount of shielded matter, the position of $R_{\mathrm{h}}$, and the relative strength of the hot and cold state viscosities at $t=t_{1}$ largely affect the model curve evolution after $t=t_{1}$. In other words, the evolving disk should not only give a good fit for the main outburst, but also end up with proper conditions at $t=t_{1}$ to reproduce the observed secondary maximum just by removing the shielding. We neglect the tidal forces and let the matter expand freely up to $R_{\text {out }} \sim 2.0 \times 10^{11} \mathrm{~cm}$ which is about the truncation radius for the assumed parameters of GS/GRS 1124-68 (Frank et al. 1992). We consider the matter passing beyond $R_{\text {out }}$ as lost from the system.

\subsection{The main outburst and the secondary maximum}

From the time $t=3.0 \mathrm{~d}$ to the onset of the secondary maximum $\left(t=t_{1}\right)$, the model disk evolves with a cold, shielded region outside the radius $R_{\mathrm{h}}$ and an X-ray irradiated hot region inside $R_{\mathrm{h}}$. For the $\mathrm{X}$-ray irradiated hot disk, the minimum critical surface densities and the $\mathrm{X}$-ray irradiation temperatures are calculated by using Eq. (10) and Eq. (8) respectively. The parameter $C$ is determined from the fits. The model $\mathrm{X}$-ray photon 


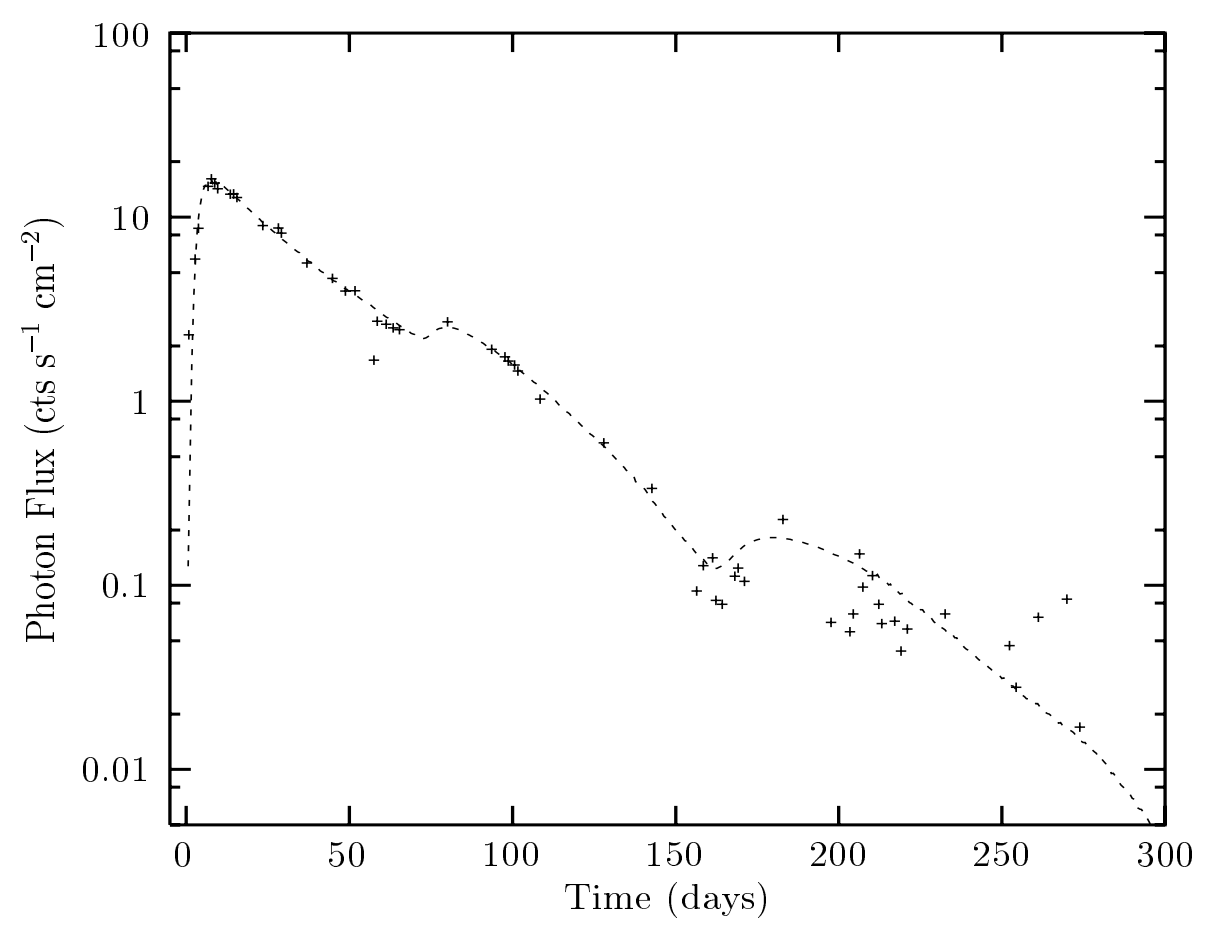

Fig. 1. Observed GINGA ASM X-ray (1-20 keV) photon flux data and the model curve (dashed line) with parameters presented in Sect. 4).

flux curve seen in Fig. 1 is obtained with $C=2.3 \times 10^{-4}$ until the onset of the bump outburst after which we expect an increase in the strength of the X-ray irradiation (see Sect. 3). With this choice of $C$, Fig. 3 shows that the hot disk radius $R_{\mathrm{h}}$ remains constant from $t=3 \mathrm{~d}$ to $t=t_{1}$. That is, the $\mathrm{X}$-ray irradiation during this period is strong enough to prevent the inward propagation of the cooling front. At $t=t_{1}$, we remove the shielding and apply the condition given by Eq. (10) to determine the maximum critical surface densities of the outermost disk with the present X-ray irradiated conditions. After the removal of the shielding, $R_{\mathrm{h}}$ at first increases and then decreases gradually governed by the decreasing strength of the X-ray irradiation (Fig. 3). A fraction of the outermost disk which has so far remained shielded and cold, makes an upward transition and the subsequent enhancement of the accretion rate from the outer disk to the inner disk gives a model curve which fits well to the observed data. For the model curve presented in Fig. 1, we chose $t_{1} \simeq 68$ days. The surface density distribution one day before the triggering of the secondary maximum is seen in Fig. 4. The removal of the shielding can be seen in the model disk scale height profile evolution calculated by using the midplane temperatures. In Fig. 2 it is clearly seen that the shielding of the outer disk $\left(R>R_{\mathrm{h}}\right)$ which is present at the early phase of the main decay is removed before the onset of the secondary maximum $\left(t \lesssim t_{1}\right.$ ) for a scattering region remaining roughly constant in size $\left(l \lesssim 10^{9} \mathrm{~cm}\right)$ during the main decay. This result is obtained by using the midplane temperatures. A full confirmation will invoke 2-D models calculating the vertical disk structure including the effect of X-ray irradiation on the inner disk at $R<R_{\mathrm{h}}$. This requires the extension of the work of Dubus et al. (2001) who calculated vertical structure for a fully irradiated disk, for the case of a disk irradiated only at $R<R_{\mathrm{h}}$ as in the present scenario.

\subsection{The bump}

Our scenario to account for the bump is based on the assumption that both the temperature and the effective scattering size of the central hot corona increases when the decreasing accretion rate of the thin inner disk leads to a decrease in the soft photon supply that cools the corona. When the truncation of the inner disk starts, the inner disk radius $R_{\text {in }}$ changes in a way that is difficult to estimate, due to uncertainties in both the strength and the functional form of the evaporation. In the present work, we take $R_{\text {in }}$ to be constant throughout the entire outburst phase, assuming that the inner disk truncation does not start immediately upon the expansion of the corona. In other words, during the bump phase, the inner thin disk underlying the corona still extends down to the last stable orbit, but it does not provide an efficient cooling for the corona any longer. To implement this idea, we simply increase the X-ray irradiation strength by a constant factor at $t=t_{2}$. This corresponds to an increase in the hot disk radius $R_{\mathrm{h}}$ (Fig. 3), and leads to an enhancement in the mass transfer rate from the outer disk. For the model curve seen in Fig. $1, t_{2}=155 \mathrm{~d}$, and the irradiation strength is increased by a factor 1.6 at $t=t_{2}$. The surface density distribution one day before the triggering of the bump is presented in Fig. 5.

The fluctuations in the observed X-ray photon flux curve of the source GS/GRS 1124-68 during the bump have small time scales, sometimes as small as a day. Variations in the inner disk radius due to the truncation of part of the inner disk and the consequent refilling in response to newly arising density gradients could be a possible reason for these fluctuations. 


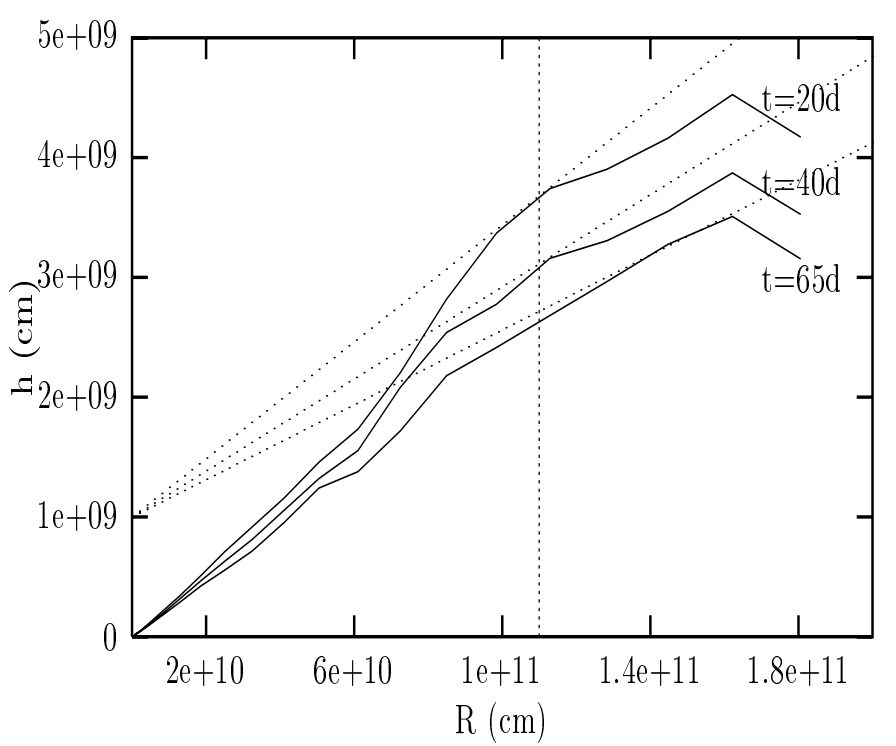

Fig. 2. Pressure scale height $h$ profiles calculated by using the midplane temperatures given by our numerical model at three different times before the onset of the secondary maximum $(t \sim 68 \mathrm{~d})$. The local fluctuations are smoothed with the same binning size for all the given curves to clarify the evolution. The vertical dotted line corresponds to $R_{\mathrm{h}}$ in our model which remains constant during the main decay. The model disk evolves in a trend to remove the shielding at about the observed secondary maximum for an inner scattering region with a size $\lesssim 10^{9} \mathrm{~cm}$. See the text for further explanation.

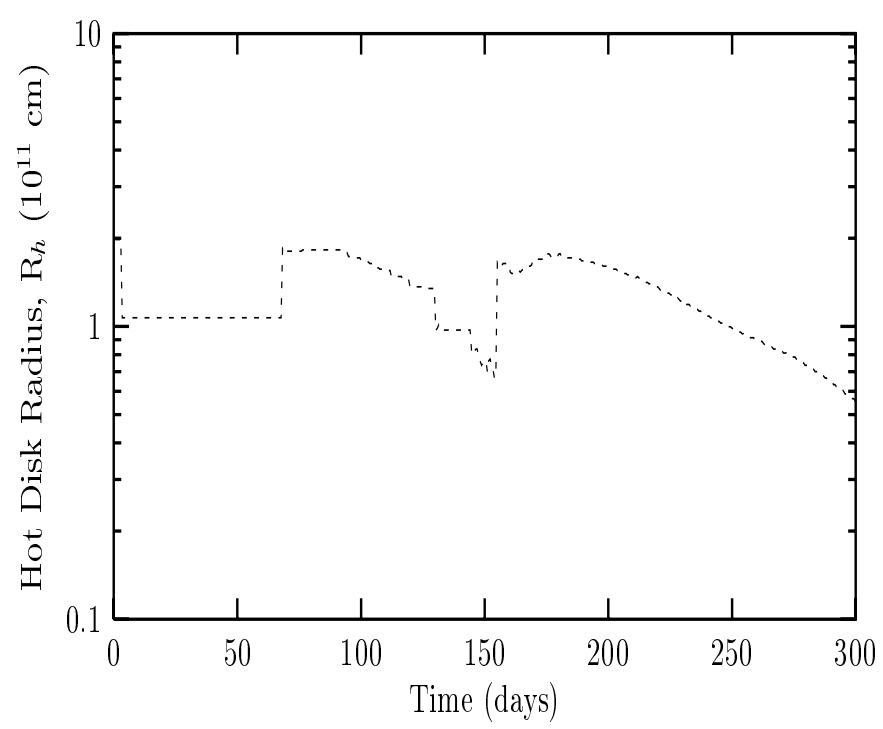

Fig. 3. Evolution of the hot disk radius $R_{\mathrm{h}}$ corresponding to the model curve seen in Fig. 1. The triggering times are $t_{1}=68 \mathrm{~d}$ for the secondary maximum and $t_{2}=155 \mathrm{~d}$ for the bump. The time intervals between the trigger and the starting of the rise in the X-ray flux are $\sim 5 \mathrm{~d}$ and $\sim 10 \mathrm{~d}$ for the secondary maximum and the bump respectively.

\subsection{The simplifications and the reliability of the numerical model}

In 2-D irradiated disk models the transitions between the hot and the cold stable states are determined by the local disk temperatures calculated by the vertical disk analyses considering the irradiation flux through the disk surface. The critical

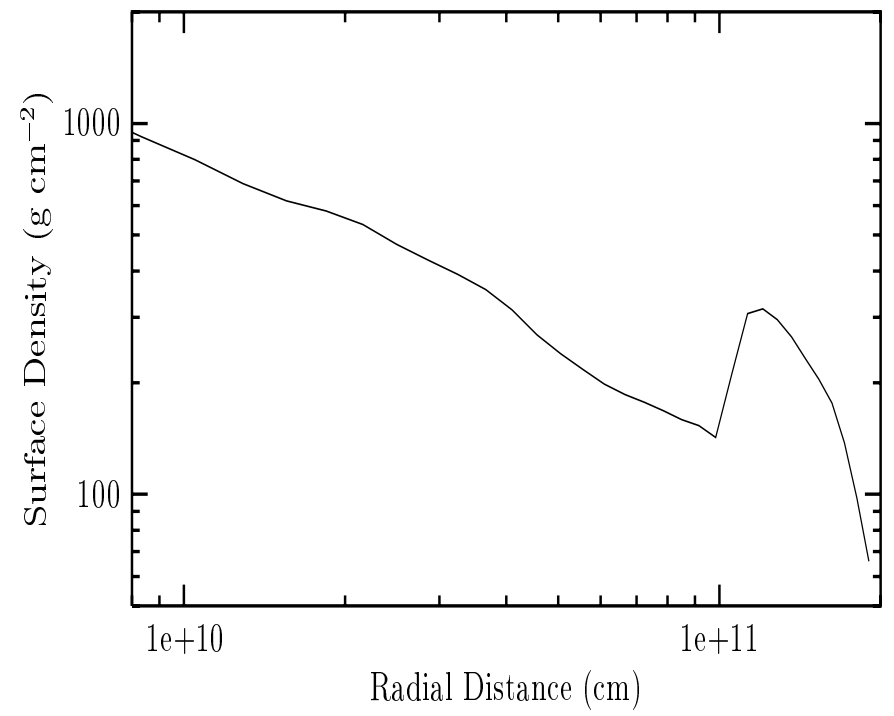

Fig. 4. Radial surface density profile one day before the onset of the secondary maximum $(t=67 \mathrm{~d}) . R_{\mathrm{h}} \sim 1 \times 10^{11} \mathrm{~cm}$.

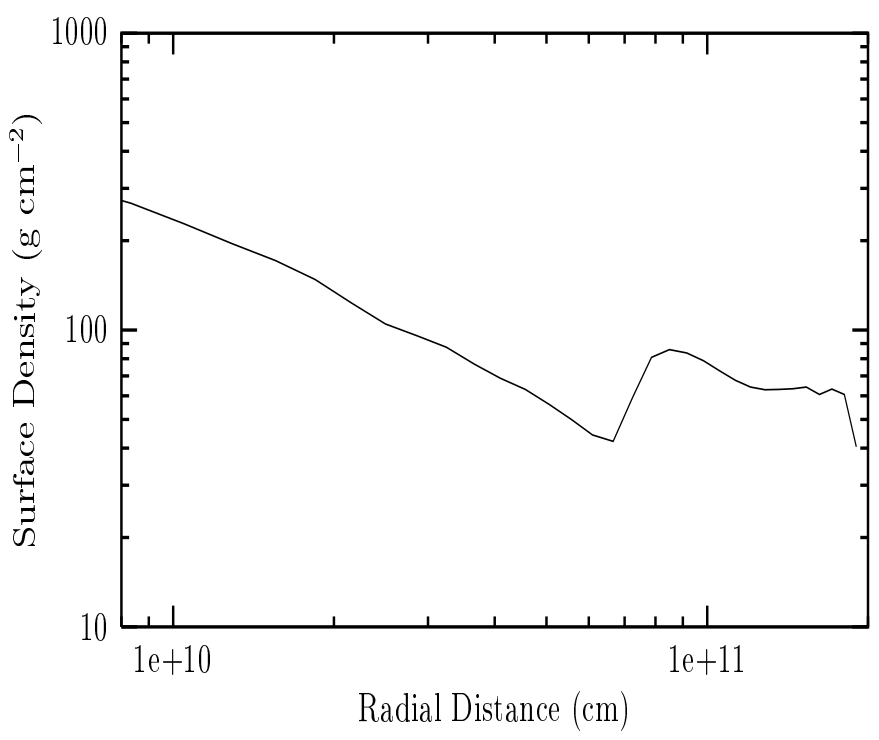

Fig. 5. Radial surface density profile one day before the onset of the bump $(t=154 \mathrm{~d}) . R_{\mathrm{h}} \sim 7 \times 10^{10} \mathrm{~cm}$.

irradiation temperatures are different for different surface densities. This becomes important in modeling the unsteady and irradiated disks. Since the irradiation temperatures are mainly determined by the conditions of the inner disk, in a 1-D disk model it is not possible to determine the critical irradiation temperatures for varying surface densities at a particular radial distance $R$. To choose a uniform critical irradiation temperature $T_{\text {irr }} \sim 10^{4} \mathrm{~K}$ may lead to erroneous results. For these reasons, in our 1-D model we use the critical surface densities for irradiated and unsteady disks calculated by Dubus et al. (2001) (DHL).

We checked the run of our numerical code using the presented results of the detailed 2-D model of DHL. The DHL model assumes a completely irradiated disk (no shielding) whereas in our numerical model for GS/GRS 1124-68 the outer disk $\left(R>R_{\mathrm{h}}\right.$ ) is assumed to be shielded during the main decay. For comparison, in an illustrative model we do not include 


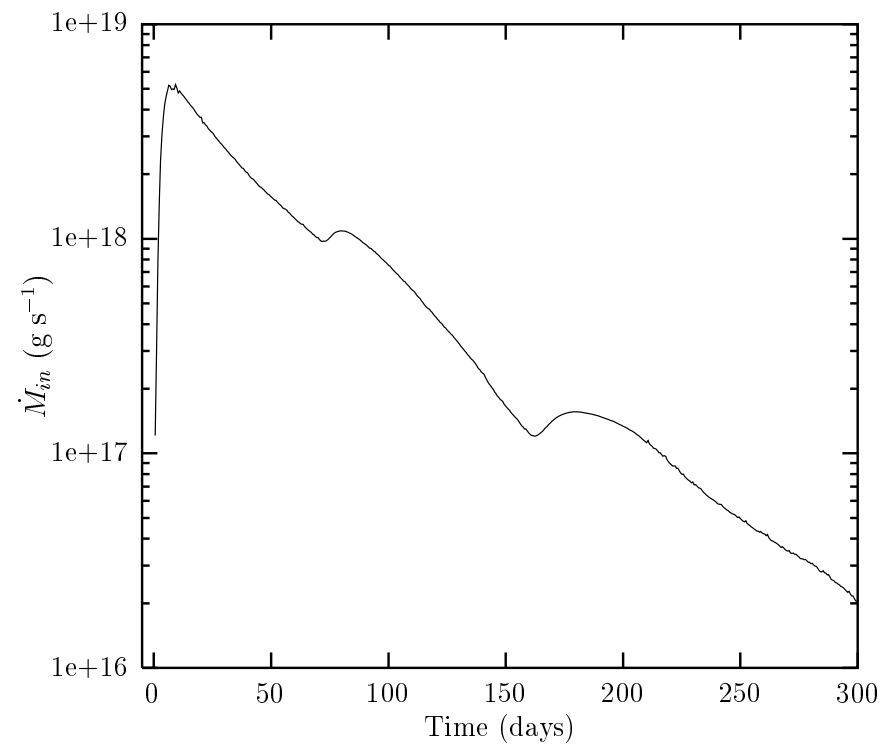

Fig. 6. Inner disk accretion rate $\dot{M}_{\text {in }}$ evolution for the model parameters presented in Sect. 4.

the shielding and adjust the amplitude of our initial Gaussian mass distribution so that the $\dot{M}_{\text {in }}$ peaks at the same level as the DHL curve. We plot this curve together with the points estimated from Fig. 6 of DHL in our Fig. 7. We also present the surface density profiles at different times of the $\dot{M}_{\text {in }}$ curve in Fig. 8. The irradiation temperatures are similar in both models. We set $\alpha_{\mathrm{h}}=0.1$ in our illustrative model, while $\alpha_{\mathrm{h}}=0.2$ in the DHL model. This is reasonable, since we employ the midplane temperatures $T_{\mathrm{c}}$ in calculating the viscosities whereas in a 2-D model effective viscosities correspond to a temperature that is between $T_{\mathrm{c}}$ and $\sim T_{\text {irr }}$ (or $T_{\text {eff }}$ ). The second difference is that the outer disk radius is determined by the tidal forces in the DHL model, whereas it is fixed and the matter going beyond this radius is assumed to be lost from the system in our illustrative model. The $\dot{M}_{\text {in }}$ and the surface density evolutions given by our illustrative model are seen to be similar to those given by DHL (our Fig. $7 \&$ Fig. 8, and Fig. 6 of DHL).

In Fig. 7 we present another $\dot{M}_{\text {in }}$ curve for a different initial mass distribution $(\Sigma \propto R)$ without changing the other parameters. This is to show that the different initial mass distributions give similar $\dot{M}_{\text {in }}$ curves, as long as most of the matter is initially located at the outer disk.

\section{Discussion and conclusions}

In general, soft X-ray transients (SXTs) show a variety of outburst light curves (Chen et al. 1997). In the present work we concentrate on the outburst light curves having a fast rise and a long lasting ( 250 days) decay behavior exhibited by the BH SXTs A0620-00, GS/GRS 1124-68, GS 2000+25 and GRO J0422+32.

We presented a new scenario to explain these outburst light curves together with the characteristic secondary maxima and the bumps seen on their decay phases. The explanations are based on the disk instability models including the effect of $\mathrm{X}$-ray irradiation, and can be summarized as follows: at the

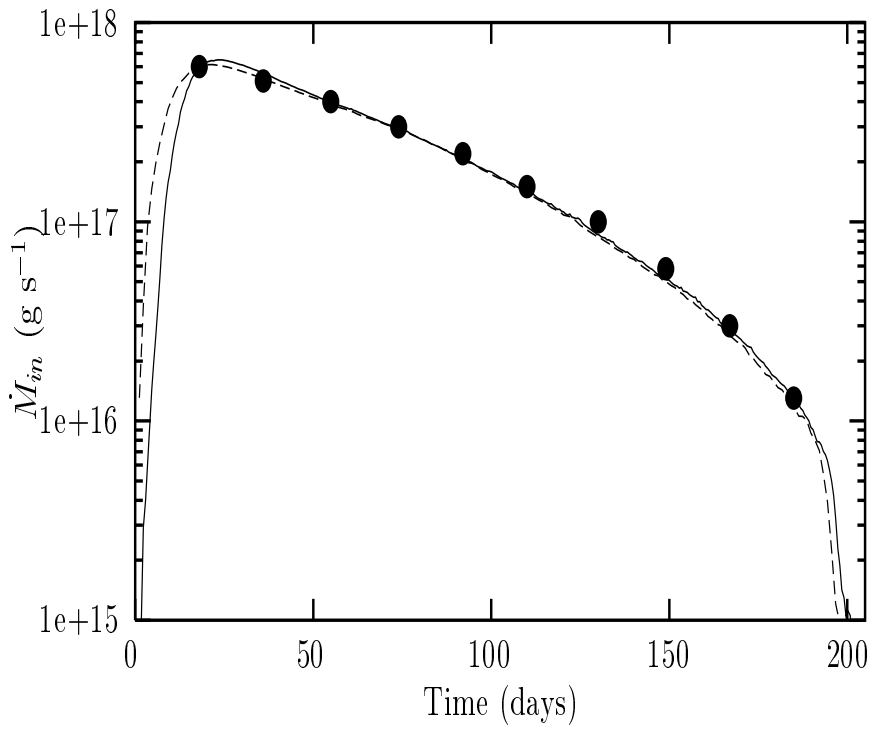

Fig. 7. Inner accretion rate evolution for the illustrative $\Sigma \propto R$ (solid curve) and Gaussian (dashed curve) initial mass distributions with the same disk parameters. The filled circles are estimated from Fig. 6 of Dubus et al. (2001) (DHL) for comparison. The amplitudes of the initial surface densities were adjusted to match our model maxima to the maximum of the DHL model. The shielding is not included in our illustrative models.

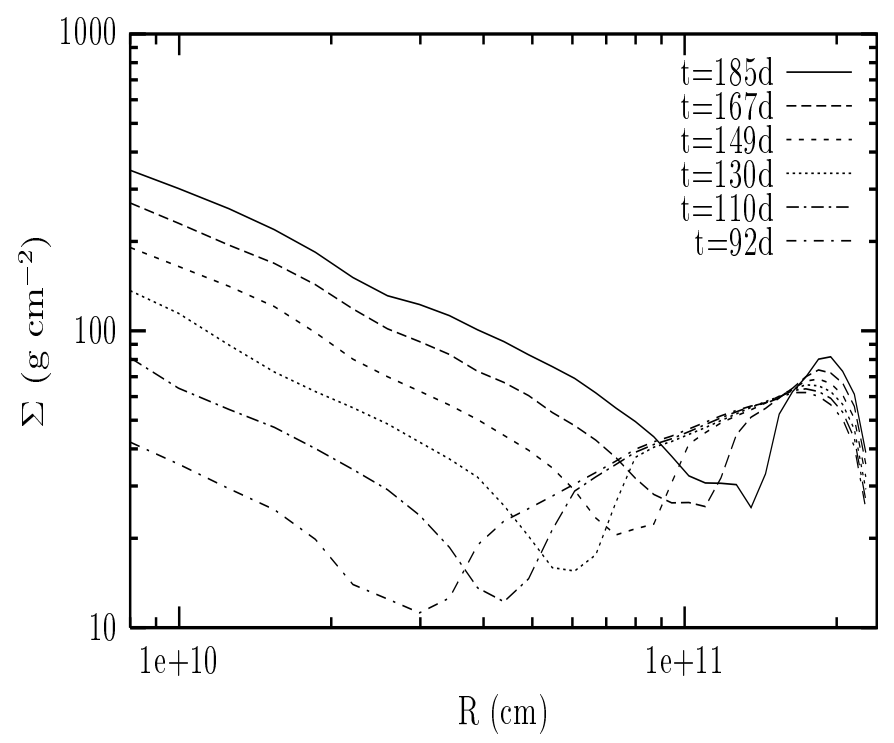

Fig. 8. Surface density distributions corresponding to different points on the $\dot{M}_{\text {in }}$ curve shown in Fig. 7 (dashed curve) given by the illustrative Gaussian initial mass distribution. These are seen to be similar to those presented in Fig. 6 of Dubus et al. (2001) for the same inner accretion rates.

beginning of the main outburst, the accumulated cold matter is released by a thermal-viscous instability. This matter expands to both lower and larger radii. Part of the outermost disk that makes a transition back to the cold state before the efficient $\mathrm{X}$-ray irradiation has started, initially remains shielded by the hot inner disk. The surface densities of the hot inner disk decrease faster than the surface densities of the shielded disk at $R>R_{\mathrm{h}}$. Finally, the shielding is removed after the pressure scale height of the disk at $R \lesssim R_{\mathrm{h}}$ has decreased enough for 
the central X-rays to illuminate the cold disk at $R>R_{\mathrm{h}}$. The consequently triggered instability outside $R_{\mathrm{h}}$ leads to enhanced mass flow resulting in the secondary maximum. The hot disk radius $R_{\mathrm{h}}$ first increases with the triggering, and later gradually decreases, governed by the decreasing irradiation flux through the outer disk. When the inner accretion rate $\dot{M}_{\text {in }}$ becomes comparable to the evaporation rate, the temperature of the corona increases due to decreasing cooling rate. Consequently, the corona which is assumed to be the source of indirect illumination heats up and expands leading to more efficient X-ray irradiation and a new thermal-viscous instability beyond the present position of $R_{\mathrm{h}}$. The subsequent enhancement of the mass transfer rate from the outer disk results in the observed bump.

We tested this scenario by using a one dimensional numerical model (Sect. 4). The model giving the X-ray photon flux curve given in Fig. 1 works briefly as follows: At the beginning of the main outburst, the accumulated matter is represented by a Gaussian mass distribution at the outer disk. The center of the Gaussian is taken to be $R=R_{0}=9 \times 10^{10} \mathrm{~cm}$. Its maximum density $\Sigma_{0}=1.1 \times 10^{3} \mathrm{~g} \mathrm{~cm}^{-2}$ is comparable to the maximum critical surface density of the cold state for the chosen parameters $M_{1}=6 M_{\odot}, \alpha_{\mathrm{c}}=0.033$ and $R_{0}=9 \times 10^{10} \mathrm{~cm}$ (Cannizzo et al. 1988; Dubus et al. 2001). We start with the hot state viscosities ( $\alpha=\alpha_{\mathrm{h}}=0.1$ ). Until the $\mathrm{X}$-ray irradiation is switched on at $t=t_{0} \mathrm{~d}$, we set $\alpha=\alpha_{\mathrm{c}}$ for the grid points with the effective temperatures $T_{\text {eff }}$ decreasing below $10000 \mathrm{~K}$, and the grid points having higher $T_{\text {eff }}$ are kept in the hot state. At $t=t_{0}=3.0 \mathrm{~d}$, the outermost cold disk region with $\alpha=\alpha_{\mathrm{c}}$ $\left(R>R_{\mathrm{h}}\right)$ is taken to be shielded. After $t=t_{0} \mathrm{~d}$, we use the critical surface densities obtained for irradiated and unsteady disks (Dubus et al. 2001). From $t=t_{0}$ to $t=t_{1}$, the disk evolves with a hot inner region $\left(R<R_{\mathrm{h}}\right)$ and a cold shielded outer region ( $R>R_{\mathrm{h}}$ ). The hot disk radius $R_{\mathrm{h}}$ is free to move, e.g. inwards, if the surface densities at the grid points inside $R_{\mathrm{h}}$ decrease below the critical minimum surface densities of the present irradiated conditions. We find that $R_{\mathrm{h}}$ remains constant until the onset of the secondary maximum $\left(t=t_{1}\right)$ for the irradiation strength $\left(C=2.3 \times 10^{-4}\right)$ that gives the best fit to the data until the onset of the bump $\left(t=t_{2}\right)$. Our 1-D numerical model does not give the irradiated vertical disk structure, so we remove the shielding at a parametrized time $t=t_{1}$ whose value, $t_{1}=68 \mathrm{~d}$, is determined from the best fits. We assume that all the disk has become irradiated at $t=t_{1}$, and the part of the outer disk which has so far remained shielded and cold makes a transition to the hot state at $t=t_{1}$. After $t=t_{1}, R_{\mathrm{h}}$ first increases by the removal of the shielding and then decreases gradually governed by the decreasing irradiation strength. To check for an indication that the shielding is removed in the 1-D model, we plot the disk thickness profiles calculated by the midplane temperatures at different times of the main decay phase. We see that the shielding of the outer disk $\left(R>R_{\mathrm{h}}\right)$ which is present during the main decay is removed at $t \sim t_{1}$ for an inner scattering region with a size $l \lesssim 10^{9} \mathrm{~cm}$. The confirmation of the initial settling and the removal of the shielding in a self-consistent model will be the subject of future work. The removal of the shielding at $t=t_{1}$ exposes the outer disk to irradiation. This leads to the secondary maximum, first in the optical, and then in the X-rays, as the enhanced mass flow reaches the inner disk. With $t_{1}=68 \mathrm{~d}$, the model reproduces the secondary maximum.

Increasing irradiation efficiency through an expanding corona, rather than removal of shielding, is taken to be the cause of the bump. At $t=t_{2}=155 \mathrm{~d}$, the irradiation temperatures are increased by a constant $(\sim 1.6)$ factor chosen to fit the amplitude of the bump. In our model, the transitions to the hot state at $t=t_{1}$ and later at $t=t_{2}$ are taken to occur simultaneously in the newly irradiated regions, rather than by the propagation of thermal fronts. The tidal forces at outer disk and the accretion from the companion are neglected. We obtained the outer disk radius $R_{\text {out }}=2 \times 10^{11} \mathrm{~cm}$ from our fits. This is about the truncation radius $R_{\mathrm{tr}} \simeq 0.9 R_{\mathrm{L}_{1}}$. The matter going beyond this radius is assumed to be lost from the system. In Sect. 4.6 we showed that the simplifications of our numerical model does not significantly affect the results.

The maximum of the outburst in X-rays is reached at $\sim 7 \mathrm{~d}$ in the model. The time interval between the triggering and the beginning of the rise given by the model is about 5 days for the secondary maximum and 10 days for the bump. These results are in good agreement with the reported delays of the X-ray maxima with respect to the optical which are $\sim 4-6$ days for the main outburst and the secondary maximum, and about two weeks for the bump (Kuulkers 1998; Ebisawa et al. 1994; Orosz et al. 1997).

We have thus shown, through a numerical model with plausible parameters (Fig. 1), that the rise and the decay of BH SXT outbursts including the secondary maximum and the bump are a simple interactive history of the effects of viscous diffusion, irradiation and hot-cold state transitions. Both the secondary maximum and the bump are events taking place, only once, in the evolution of the disk after the main outburst.

The observed fluctuations shown during the bump could be due to the small variations of the inner disk radius when the evaporation rate becomes comparable to the inner disk accretion rate. The evaporation is expected to be strongest at the inner disk and steeply decrease with increasing radius (Meyer et al. 2000; Dubus et al. 2001). Then the large density gradients due to an evacuation of the inner disk by the evaporation could result in the variations of the inner accretion rate, which could easily modify the X-ray flux. In our numerical model, we do not address these fluctuations. A reasonable fit to what might plausibly be the mean behavior of the bump is produced for GRS/GS 1124-68.

In our numerical model we do not include possible mass losses from the disk surface due to evaporation (Meyer et al. 2000; Dubus et al. 2001; Shaviv et al. 1999). The X-ray irradiation is expected to increase the wind losses from the outer disk (de Kool \& Wickramasinghe 1999). Although there is no general agreement on the physics of evaporation, it has probably no significant effect on the outburst light curves (Dubus et al. 2001). However, there is a consensus that evaporation is important in the quiescent states, and the inner disk is probably truncated because of the resultant mass losses. Meyer-Hofmeister \& Meyer (1999) and Dubus et al. (2001) obtain the long outburst recurrence times of SXTs ( few ten years or more) by including evaporation in their numerical model. 
Acknowledgements. We acknowledge support from the BDP Doctoral Research program of TÜBITAK (The Scientific and Technical Research Council of Turkey) and through The High Energy Astrophysics Research Group TBAG-Ç-4 of TÜBITAK. We thank S. Kitamoto for providing GINGA ASM outburst data for the source GS/GRS 1124-68. Part of this research was done during the Summer Research Semester in Astrophysics (2001) of the Feza Gürsey Institute of TÜBITAK. MAA acknowledges support from the Turkish Academy of Sciences. We thank the referee for useful criticism.

\section{References}

Alexander, D. R., \& Ferguson, J. W. 1994, ApJ, 437, 879

Cannizzo, J. K. 1992, Accretion Disks in Compact Stellar Systems, ed. J. C. Wheeler, 6

Cannizzo, J. K., Shafter, A. W., \& Wheeler, J. C. 1988, ApJ, 333, 227

Cannizzo, J. K., Wheeler, J. C., \& Gosh, P. 1985, in Proc. Cambridge Workshop on Cataclysmic Variables and Low Mass X-ray Binaries, ed. D. Q. Lamb, \& J. Patterson

Cannizzo, J. K. 1998, ApJ, 494, 366

Cannizzo, J. K. 2000, ApJ, 534, L35

Chen, W., Livio, M., \& Gehrels, N. 1993, ApJ, 408, L5

Chen, W., Shrader, C. R., \& Livio, M. 1997, ApJ, 491, 312

Czerny, M., \& King, A. R. 1989, MNRAS, 236, 843

de Jong, J. A., van Paradijs, J., \& Augusteijn, T. 1996, A\&A, 314, 484

de Kool, M., \& Wickramasinghe, D. 1999, MNRAS, 307, 449

Dubus, G., Lasota, J.-P., Hameury, J.-M., \& Charles, P. 1999, MNRAS, 303, 139

Dubus, G., Hameury, J.-M., \& Lasota, J.-P. 2001, A\&A, 373, 251

Ebisawa, K., Ogawa, M., Aoki, T., et al. 1994, PASJ, 46, 375

Ertan, Ü., \& Alpar, M. A. 1998, A\&A, 336, 220
Frank, J., King, A. R., \& Raine, D. 1992, Accretion Power in Astrophysics (Cambridge: Cambridge University Press)

Hameury, J.-M., Menou, K., Dubus, G., Lasota, J.-P., \& Huré, J.-M., 1998, MNRAS, 298, 1048

Haswell, C. A., King, A. R., Murray, J. R., \& Charles, P. A. 2001, MNRAS, 321, 475

Hōshi, R. 1979, Progr. Theor. Phys., 61, 1307

Huang, M., \& Wheeler, J. C. 1989, ApJ, 343, 229

King, A. R. 1989, MNRAS, 241, 365

King, A. R. 1998, MNRAS, 296, L45

King, A. R., \& Ritter, H. 1998, MNRAS, 293, L42

Kuulkers, E. 1998, New Astron. Rev., 42, 1

Ludwig, K., Meyer-Hofmeister, E., \& Ritter, H. 1994, A\&A, 290, 473

Lynden-Bell, D., \& Pringle, J. E. 1974, MNRAS, 168, 603

McClintock, J. E., Bailyn, C. D., \& Remillard, R. A. 1992, IAU Circ., 5499

Meyer, F., \& Meyer-Hofmeister, E. 1984, A\&A, 140, L35

Meyer, F., Liu, B. F., \& Meyer-Hofmeister, E. 2000, A\&A, 361, 175

Meyer, F., \& Meyer-Hofmeister, E. 1981, A\&A, 104, L10

Meyer-Hofmeister, E., \& Meyer, F. 1999, A\&A, 348, 154

Mineshige, S., \& Wheeler, J. C. 1989, ApJ, 343, 241

Mineshige, S. 1994, ApJ, 431, L99

Orosz, J. A., Remillard, R. A., Bailyn, C. D., \& McClintock, J. E. 1997, ApJ, 478, L830

Osaki, Y. 1974, PASJ, 26, 420

Piran, T. 1978, ApJ, 221, 652

Shafter, A. W., Wheeler, J. C., \& Cannizzo, J. K. 1986, ApJ, 305, 261

Shakura, N. I., \& Sunyaev, R. A. 1973, A\&A, 24, 337

Shaviv, G., \& Wehrse, R. 1986, A\&A, 159, L5

Shaviv, G., Wickramasinghe, D., \& Wehrse, R. 1999, A\&A, 344, 639

Tanaka, Y., \& Shibazaki, N. 1996, ARA\&A, 34

Tuchman, Y., Mineshige, S., \& Wheeler, J. C. 1990, ApJ, 359, 164

van Paradijs, J., \& McClintock, J. E. 1994, A\&A, 290, 133

West, R. M. 1991, Proc. Workshop Nova Muscae, Lyngby, ed. S. Brandt, 143 